\title{
AN ORTHOGONALLY INVARIANT MINIMAX ESTIMATOR OF THE COVARIANCE MATRIX OF \\ A MULTIVARIATE NORMAL POPULATION
}

\author{
By
}

\author{
Akimichi TAKEMURA
}

\begin{abstract}
In the problem of estimating the covariance matrix of a multivariate normal population James and Stein (1961) obtained a minimax estimator by considering the best invariant estimator with respect to the triangular group. In this paper we propose an orthogonally invariant estimator obtained by averaging the minimax estimator with respect to the invariant measure on the orthogonal group. Explicit forms of the proposed estimator are given for dimensions 2 and 3. Risk is evaluated for various population covariance matrices and it shows a substantial improvement over the minimax estimator for a wide range of population covariance matrices.
\end{abstract}

\section{$\S 1$. Introduction.}

In the problem of estimating the covariance matrix of a multivariate normal population the usual estimator is the sample covariance matrix $S=A / n$ where $A$ is distributed according to the Wishart distribution $\mathscr{W}(\Sigma, n)$. Although $S$ is unbiased it is known that the (sample) characteristic roots of $S$ tend to be more spread out than the corresponding (population) roots of $\Sigma$. This can be seen as follows. Let $\lambda_{1}$ be the largest characteristic root of $\Sigma$ and $\xi$ be an associated unit characteristic vector, then $\xi^{\prime} S \xi$ is unbiased for $\lambda_{1}$. Let $l_{1}$ be the largest sample root, then

$$
\mathcal{E}\left(l_{1}\right)=\mathcal{E}\left(\max _{x^{\prime} x=1} x^{\prime} S x\right) \geqq \mathcal{E}\left(\xi^{\prime} S \xi\right)=\lambda_{1} .
$$

See Van der Vaart (1961), Anderson (1963). Similarly for the smallest root $\lambda_{p}$, $l_{p}$ of $\Sigma, S$, respectively, we have $\mathcal{E}\left(l_{p}\right) \leqq \lambda_{p}$. It is these implicit maximization and minimization processes for each observed $S$ that makes the sample roots more spread out than the population roots. Actually in terms of majorization the following holds: $\left(\mathcal{E}\left(l_{1}\right), \cdots, \mathcal{E}\left(l_{p}\right)\right)$ majorizes $\left(\lambda_{1}, \cdots, \lambda_{p}\right)$. See Chapter 12 , Section $\mathrm{E}$ of Marshall and Olkin (1979).

Received July 27, 1983. Revised February 1, 1984. 
The above consideration suggests that we shrink the sample roots toward a middle value. This is analogous to the Stein-type estimation of a multivariate normal mean vector. Earlier works along this direction can be found in Stein (1975), Efron and Morris (1976), Haff (1977, 1979a, b, 1980), Eaton (1970), Sugiura and Fujimoto (1982) and others.

Another approach was taken in Stein (1956), James and Stein (1961), Selliah (1964), and Olkin and Selliah (1977). They are concerned with minimax estimation of the covariance matrix. Minimax estimators can be obtained by considering the best invariant estimator with respect to the triangular group $G_{T}^{+}$(the group consisting of lower triangular matrices with positive diagonal elements). An unappealing property of these estimators is that they depend on the coordinate system.

In this paper we propose an orthogonally invariant minimax estimator which is derived from the minimax estimators above by averaging them. The idea of averaging already appears in Stein (1956) and the specific estimator proposed below is briefly mentioned in Eaton (1970) (his formula 3.6). But it seems to have never been studied carefully.

In Section 2 we derive the estimator and study its properties. Details of computation are given in Section 4 For dimensions 2 and 3 the estimator is given explicitly. For larger dimensionalities the explicit integration involved seems formidable. The Monte Carlo method is always available, but some good approximation is desirable. In Section 3 we study the risk behavior of the estimator. If the number of degrees of freedom is not too large compared to the dimensionality, it shows a substantial improvement over the minimax estimator mentioned above for a wide range of population covariance matrices.

\section{$\S 2$. Derivation of the estimator.}

Consider the problem of estimating $\Sigma$ with the following loss functions:

$$
\begin{aligned}
& L_{1}(\Sigma, \hat{\Sigma})=\operatorname{tr}\left(\hat{\Sigma} \Sigma^{-1}\right)-\log \operatorname{det}\left(\hat{\Sigma} \Sigma^{-1}\right)-p, \\
& L_{2}(\Sigma, \hat{\Sigma})=\operatorname{tr}\left(\hat{\Sigma} \Sigma^{-1}-I\right)^{2} .
\end{aligned}
$$

The best scalar multiples of $A$ for these loss functions have constant risk, but they are not minimax. Minimax estimators were obtained by considering the best invariant estimator with respect to $G_{T}^{+}$. They are of the form

$$
\hat{\Sigma}(A)=T D T^{\prime},
$$

where $D=\operatorname{diag}\left(d_{1}, \cdots, d_{p}\right)$ and $T \in G_{T}^{+}$with $T T^{\prime}=A$.

For $L_{1}$ 


$$
d_{i}=\frac{1}{n+p+1-2 i}, \quad i=1, \cdots, p .
$$

See Stein (1956), James and Stein (1961). For $L_{2}$ see Selliah (1964), Olkin and Selliah (1977).

Note that for $L_{1}$ we have $d_{1}<\cdots<d_{p}$. The same ordering seems to hold for $L_{2}$ as well. This causes a rather unpleasant asymmetry of the estimator: the first few rows and columns become smaller compared to others. This asymmetry leads to the idea of symmetrizing the estimator by averaging over different coordinates. Let $\Gamma$ be an orthogonal matrix corresponding to a change of orthonormal basis. Applying (2.2) in the new coordinate system we obtain $\hat{\Sigma}_{\Gamma}=$ $\Gamma T_{\Gamma} D T_{\Gamma}^{\prime} \Gamma^{\prime}$ where $T_{\Gamma} T_{\Gamma}^{\prime}=\Gamma^{\prime} A \Gamma . \hat{\Sigma}_{\Gamma}$ has the same constant risk as $\hat{\Sigma}$. Now let $\mu$ be a probability distribution on $O(p)$, the group of $p \times p$ orthogonal matrices. An improved estimator can be obtained by averaging:

$$
\hat{\Sigma}_{\mu}(A)=\int_{O(p)} \Gamma T_{\Gamma} D T_{\Gamma}^{\prime} \Gamma^{\prime} d \mu(\Gamma) .
$$

Note that $\hat{\Sigma}_{\mu}$ is minimax being an improvement over a minimax estimator. Interesting cases are (i) $\mu$ : the uniform distribution of permutation matrices, (ii) $\mu$ : the uniform distribution (Haar measure) on $O(p)$. These cases are briefly mentioned in Stein (1956, formula 4.13), Eaton (1970, formula 3.6), respectively. See Sharma (1980) too.

For this paper we consider the uniform distribution on $O(p)$ and study the resulting estimator:

$$
\hat{\Sigma}_{0}(A)=\int_{O(p)} \Gamma T_{\Gamma} D T_{\Gamma}^{\prime} \Gamma^{\prime} d \Gamma
$$

Note that $\hat{\Sigma}_{0}(A)$ is orthogonally invariant. Hence $\hat{\Sigma}_{0}$ modifies only the characteristic roots of $A$. Define $\phi_{1}, \cdots, \phi_{p}$ by

$$
\operatorname{diag}\left(\phi_{1}(\alpha), \cdots, \phi_{p}(\alpha)\right)=\hat{\Sigma}_{0}\left(\operatorname{diag}\left(\alpha_{1}, \cdots, \alpha_{p}\right)\right),
$$

where $\alpha=\left(\alpha_{1}, \cdots, \alpha_{p}\right)$. We are interested in the behavior of $\phi_{1}, \cdots, \phi_{p}$. We will see the shrinking of the roots mentioned in the introduction. Let us look at the simplest case $p=2$.

THEOREM 2.1. For $p=2$

$$
\begin{aligned}
& \phi_{1}\left(\alpha_{1}, \alpha_{2}\right)=\alpha_{1} c_{1}=\alpha_{1}\left(\frac{\sqrt{\alpha_{1}}}{\sqrt{\alpha_{1}}+\sqrt{\alpha_{2}}} d_{1}+\frac{\sqrt{\alpha_{2}}}{\sqrt{\alpha_{1}}+\sqrt{\alpha_{2}}} d_{2}\right), \\
& \phi_{2}\left(\alpha_{1}, \alpha_{2}\right)=\alpha_{2} c_{2}=\alpha_{2}\left(\frac{\sqrt{\alpha_{2}}}{\sqrt{\alpha_{1}}+\sqrt{\alpha_{2}}} d_{1}+\frac{\sqrt{\alpha_{1}}}{\sqrt{\alpha_{1}}+\sqrt{\alpha_{2}}} d_{2}\right) .
\end{aligned}
$$


Note that as $\alpha_{1} / \alpha_{2}$ approaches $\infty, \phi_{1} \sim \alpha_{1} d_{1}, \phi_{2} \sim \alpha_{2} d_{2}$. Now for $L_{1}$ we have $d_{1}=1 /(n+1)<1 / n<d_{2}=1 /(n-1)$. This shows that if $\alpha_{1} \gg \alpha_{2}$ then the larger root is shrunk and the smaller root is expanded relative to the unbiased case $S=A / n$. When $\alpha_{1}=\alpha_{2}=\alpha$ then $\phi_{1}=\phi_{2}=\alpha\left(d_{1}+d_{2}\right) / 2$. The shrinking factors $c_{1}, c_{2}$ change smoothly between these two cases.

For $p=3$ the integration over $O(3)$ is already tedious. We give an infinite series expression for $\phi_{1}, \phi_{2}, \phi_{3}$. Convergence is reasonably fast but the form of the series is not very revealing. Let $(a)_{k}=a(a+1) \cdots(a+k-1)$ and let

$$
F_{1}\left(a ; b, b^{\prime} ; c ; x, y\right)=\sum_{m=0}^{\infty} \sum_{n=0}^{\infty} \frac{(a)_{m+n}(b)_{m}\left(b^{\prime}\right)_{n}}{m ! n !(c)_{m+n}} x^{m} y^{n}
$$

be Appell's hypergeometric function of two variables. Furthermore for convenience let $H\left(b, b^{\prime} ; c ; x, y\right)=F_{1}\left(1 ; b / 2, b^{\prime} / 2 ; c / 2 ; x, y\right)$.

THEOREM 2.2. Let $p=3$ and $0 \leqq \alpha \leqq \beta<1$. Then

$$
\begin{aligned}
& \phi_{1}(1,1-\alpha, 1-\beta) \\
&= \frac{d_{1}+d_{2}+d_{3}}{3}-\frac{\left(d_{3}-d_{1}\right)(\alpha+\beta)}{15}-\frac{d_{2}-d_{1}}{3} H(1,1 ; 5 ; \alpha, \beta) \\
&-\frac{d_{3}-d_{2}}{105(1-\alpha)}\left\{(\alpha-\beta)^{2} H(3 ; 3 ; 9 ; \beta, \gamma)+3 \alpha^{2}(1-\beta) H(5 ; 3 ; 9 ; \beta, \gamma)\right. \\
&\left.\quad+3 \beta^{2}(1-\alpha) H(5,1 ; 9 ; \beta, \gamma)\right\},
\end{aligned}
$$

where $\gamma=(\beta-\alpha) /(1-\alpha)$,

$$
\begin{aligned}
& \phi_{2}(1,1-\alpha, 1-\beta) \\
& =\frac{d_{1}+d_{2}+d_{3}}{3}-\frac{\alpha}{15}\left(7 d_{1}+5 d_{2}+3 d_{3}\right)-\frac{\beta}{15}\left(d_{3}-d_{1}\right) \\
& -\frac{d_{2}-d_{1}}{105}\left\{3 \alpha^{2} H(3,1 ; 9 ; \alpha, \beta)+2 \alpha(\alpha-\beta) H(3,3 ; 9 ; \alpha, \beta)\right. \\
& \left.+3(\alpha-\beta)^{2} H(3,5 ; 9 ; \alpha, \beta)\right\} \\
& -\frac{d_{3}-d_{2}}{105(1-\alpha)}\left\{3(\alpha-\beta)^{2} H(1,5 ; 9 ; \beta, \gamma)+3 \alpha^{2}(1-\beta) H(3,5 ; 9 ; \beta, \gamma)\right. \\
& \left.+\beta^{2}(1-\alpha) H(3,3 ; 9 ; \beta, \gamma)\right\} \\
& \phi_{3}(1,1-\alpha, 1-\beta)=(1-\beta)\left\{d_{1}+d_{2}+d_{3}-\phi_{1}-\phi_{2} /(1-\alpha)\right\} \text {. }
\end{aligned}
$$

Note that these formulas suffice for all cases because the estimator is invariant with respect to scale change and permutation of roots.

Although it is difficult to see from these expressions, the shrinking of the roots becomes apparent when values of $\left(\phi_{1}, \phi_{2}, \phi_{3}\right)$ are computed for various choices of $\alpha$ and $\beta$. 
For general dimension $p, p(p-1) / 2$-fold integration is involved. Although infinite series expression as in Theorem 2.2 is always possible in principle, it will be complicated and convergence might be slow. Then a Monte Carlo method can be used. We will discuss this in Section 3 and Section 4 . Here we give a qualitative description of the estimator. Note that $D$ can be written as $D=$ $d_{1} E_{11}+\cdots+d_{p} E_{p p}$ where $E_{i i}$ has 1 in $(i, i)$-th position and 0 everywhere else. Putting this into (2.5) we see that $\phi_{1}, \cdots, \phi_{p}$ are linear in $d_{1}, \cdots, d_{p}$. Therefore we can write

$$
\phi_{i}(\alpha)=\alpha_{i} c_{i}=\alpha_{i}\left(w_{i 1} d_{1}+\cdots+w_{i p} d_{p}\right), \quad i=1, \cdots, p .
$$

Let $W(\boldsymbol{\alpha})=\left(w_{i j}(\boldsymbol{\alpha})\right)$.

THEOREM 2.3. $W(\alpha)$ is doubly stochastic, namely $w_{i j} \geqq 0, \sum_{j} w_{i j}=1, \sum_{i} w_{i j}=1$.

Proof. It is easy to show that

$$
\alpha_{i} w_{i j}=\int\left(\Gamma T_{\Gamma}\right)_{i j}^{2} d \Gamma .
$$

Hence $w_{i j} \geqq 0$. Considering the special case $D=I$ proves $w_{i 1}+\cdots+w_{i p}=1, i=$ $1, \cdots, p . \quad \sum_{i} w_{i j}=1$ is a consequence of the following lemma.

LEMMA 2.1.

$$
\operatorname{tr}\left(\hat{\Sigma}_{0}(A) A^{-1}\right)=\operatorname{tr} D
$$

Proof is straightforward and omitted.

\section{§3. Risk.}

For $L_{1}$ the risk can be evaluated in the following fairly simple form and gives a nice qualitative understanding of its behavior.

THEOREM 3.1.

$$
\begin{aligned}
R_{1}\left(\Sigma, \hat{\Sigma}_{0}\right) & =\mathcal{E}\left\{L_{1}\left(\Sigma, \hat{\Sigma}_{0}(A)\right)\right\} \\
& =-\sum_{i=1}^{p} \mathcal{E} \log c_{i}(\alpha)-p \log 2-\sum_{i=1}^{p} \psi\left(\frac{n+1-i}{2}\right) .
\end{aligned}
$$

where $c_{i}(\alpha)$ 's are the shrinking factors and $\phi(a)=\Gamma^{\prime}(a) / \Gamma(a)$.

Proof. We look at $\operatorname{tr} \Sigma^{-1} \hat{\Sigma}$ term first.

$$
\mathcal{E}_{\Sigma}\left\{\operatorname{tr} \Sigma^{-1} \hat{\Sigma}_{0}\right\}=\mathcal{E}_{\Sigma}\left(\operatorname{tr} \Sigma^{-1} \int \Gamma T_{\Gamma} D T_{\Gamma}^{\prime} \Gamma^{\prime} d \Gamma\right)
$$




$$
\begin{aligned}
& =\int \mathcal{E}_{\Sigma^{*}}\left(\operatorname{tr} \Sigma^{*-1} T D T^{\prime}\right) d \Gamma \\
& =\int \mathcal{E}_{I}\left(\operatorname{tr} T^{\prime} T D\right) d \Gamma \\
& =\sum_{i=1}^{p} d_{i} \mathcal{E} \chi_{n+p-2 i+1}^{2} \\
& =p .
\end{aligned}
$$

where $\Sigma^{*}=\Gamma^{\prime} \Sigma \Gamma$. The rest of the proof is straightforward.

COROLLARY 3.1 .

$$
\sum \log d_{i} \leqq \Sigma \log c_{i} \leqq p \log \left(\Sigma d_{i} / p\right)
$$

hence

$$
\begin{gathered}
-p \log \left(\frac{\sum d_{i}}{p}\right)-p \log 2-\Sigma \phi\left(\frac{n+1-i}{2}\right) \leqq R_{1}\left(\Sigma, \hat{\Sigma}_{0}\right) \\
\leqq-\Sigma \log d_{i}-p \log 2-\Sigma \phi\left(\frac{n+1-i}{2}\right)
\end{gathered}
$$

ProOF. We use the concavity of $\log$ and Jensen's inequality.

$$
\frac{1}{p} \Sigma \log c_{i} \leqq \log \left(\Sigma c_{i} / p\right)=\log \left(\Sigma d_{i} / p\right) \text {. }
$$

This proves the second inequality of (3.3), Now

$$
\log c_{i}=\log \left(w_{i 1} d_{1}+\cdots+w_{i p} d_{p}\right) \geqq \sum_{j} w_{i j} \log d_{i} .
$$

Adding over different $i$ we obtain

$$
\sum_{i} \log c_{i} \geqq \sum_{i, j} w_{i j} \log d_{j}=\sum_{j} \log d_{j}
$$

This proves the first inequality.

It can be easily shown that the right hand side of [3.4) is the minimax risk: $R_{1}\left(\Sigma, T D T^{\prime}\right)$. The left hand side of (3.4) gives an absolute bound for the improvement by using $\hat{\Sigma}_{0}$. This bound is attained if (3.5) holds with equality, i.e. if $c_{1}=\cdots=c_{p}$. This happens when $\alpha_{1}=\cdots=\alpha_{p}$. Therefore we expect that the largest improvement occurs when $\Sigma=I$. Now since $\hat{\Sigma}_{0}$ is minimax, its risk has to approach the minimax risk for some $\Sigma$. This corresponds to having the equality in (3.6) for all $i$. This implies that $W(\alpha)$ in Theorem 2,3 is a permutation matrix. In the 2-dimensional case this happens when $\alpha_{1} / \alpha_{2} \rightarrow \infty$. In general dimensions it is not easy to say when $W(\alpha)$ approaches a permutation matrix but we expect that it corresponds to the case of extreme singularity of $A$. Here we 
present some Monte Carlo results to illustrate these points.

First consider the case $\Sigma=I$. For $p=2$ and $p=5$ and for selected values of $n$ we list risk of $S$, minimax risk, risk of $\hat{\Sigma}_{0}$, and the lower bound given in (3.4). The number in parentheses after the minimax risk is its percentage to the risk of $S$. The other numbers in parentheses are percentages to the minimax risk.

\begin{tabular}{rcccc}
\multicolumn{5}{c}{$p=2$} \\
$n$ & $R_{1}(S)$ & minimax & $R_{1}\left(\hat{\Sigma}_{0}\right)$ & lower $b d$. \\
2 & 2.54 & $2.25(88.7)$ & $2.07(91.8)$ & $1.97(87.2)$ \\
3 & 1.35 & $1.23(91.3)$ & $1.14(92.5)$ & $1.12(90.5)$ \\
4 & .927 & $.862(93.0)$ & $.808(93.7)$ & $.798(92.5)$ \\
6 & .571 & $.543(95.1)$ & $.518(95.3)$ & $.515(94.8)$ \\
10 & .324 & $.314(96.9)$ & $.304(97.0)$ & $.304(96.8)$ \\
15 & .210 & $.206(97.9)$ & $.202(97.9)$ & $.201(97.8)$
\end{tabular}

\begin{tabular}{rllll}
\multicolumn{5}{c}{$p=5$} \\
$n$ & $R_{1}(S)$ & minimax & $R_{1}\left(\hat{\Sigma}_{0}\right)$ & lower $b d$. \\
5 & 5.96 & $4.76(79.9)$ & $3.9(82)$ & $3.06(64.2)$ \\
6 & 3.99 & $3.28(82.3)$ & $2.73(83.2)$ & $2.41(73.5)$ \\
8 & 2.52 & $2.17(86.0)$ & $1.88(86.4)$ & $1.78(82.0)$ \\
10 & 1.87 & $1.65(88.5)$ & $1.47(88.7)$ & $1.43(86.2)$ \\
15 & 1.14 & $1.05(92.0)$ & $.970(92.1)$ & $.959(91.1)$
\end{tabular}

The following serves as a concise summary: (when $\Sigma=I$ ) the ratio of the risk of $\hat{\Sigma}_{0}$ to the minimax risk is roughly equal to the ratio of the minimax risk to the risk of $S$. Also note that the absolute lower bound is realistic for $n$ not too close to $p$. These observations hold in our other Monte Carlo results as well.

The remaining question is how $\Sigma$ should be close to being singular for the risk to approach the minimax risk.

For $p=2$, the risk depends only on the ratio of two population roots, say, $\lambda=\lambda_{1} / \lambda_{2}\left(\lambda_{1} \leqq \lambda_{2}\right)$. The following table gives values of the risk for $n=2,7, \lambda^{1 / 4}$ $=.2, .4, \cdots, 1.0$. $\%$ means percentage of the risk to the minimax risk.

\begin{tabular}{cccccc}
\multicolumn{3}{c}{$n=2$} & \multicolumn{3}{c}{$n=7$} \\
$\lambda$ & risk & $\%$ & $\lambda$ & risk & $\%$ \\
1.0000 & 2.069 & 91.8 & 1.0000 & 0.4400 & 95.9 \\
0.4096 & 2.076 & 92.1 & 0.4096 & 0.4409 & 96.1 \\
0.1296 & 2.100 & 93.2 & 0.1296 & 0.4439 & 96.7 \\
0.0256 & 2.147 & 95.3 & 0.0256 & 0.4496 & 97.9 \\
0.0016 & 2.212 & 98.2 & 0.0016 & 0.4559 & 99.3
\end{tabular}


We see that the risk approaches the minimax risk only when $\Sigma$ is very close to singular. This is a real advantage of using $\hat{\Sigma}_{0}$. From our other Monte Carlo results the above seems to hold for general $p$, namely, $\hat{\Sigma}_{0}$ is a substantial improvement over the constant risk minimax estimator for wide range of population covariance matrices.

\section{$\S 4$. Proofs and some computational details.}

We are going to give some details of the derivation of Theorems 2.1 and 2.2 . First we note the following.

LEMMA 4.1.

$$
\hat{\Sigma}_{0}(A)=2 \int_{|\Gamma|=1} \Gamma T_{\Gamma} D T_{\Gamma}^{\prime} \Gamma^{\prime} d \Gamma
$$

Proof of TheOREM 2.1. By Lemma 4.1 we can represent the uniform distribution on $O(2)$ by

$$
\left(\begin{array}{rr}
\cos \theta & -\sin \theta \\
\sin \theta & \cos \theta
\end{array}\right)
$$

where $\theta$ is uniformly distributed on $[0,2 \pi]$. Since $W$ is doubly stochastic we only need to calculate $w_{22}\left(\alpha_{1}, \alpha_{2}\right)=w_{22}\left(\alpha_{1} / \alpha_{2}, 1\right)$. Let $\alpha=\alpha_{1} / \alpha_{2}$ and $A=\operatorname{diag}(\alpha, 1)$. Then by (2.12)

$$
w_{22}=\int\left(\Gamma T_{\Gamma}\right)_{22}^{2} d \Gamma=\int \Gamma_{22}^{2} T_{\Gamma 22}^{2} d \Gamma
$$

Let $\Gamma^{\prime}$ be given by (4.2). Then it is easy to obtain $T_{\Gamma 22}=\sqrt{\alpha} / \sqrt{\alpha \cos ^{2} \theta+\sin ^{2} \theta}$. Hence

$$
\begin{aligned}
w_{22} & =\frac{1}{2 \pi} \int_{0}^{2 \pi} \frac{\alpha \cos ^{2} \theta}{\alpha \cos ^{2} \theta+\sin ^{2} \theta} d \theta \\
& =\frac{\sqrt{\alpha}}{\sqrt{\alpha+1}} .
\end{aligned}
$$

Proof of Theorem 2.2. We show only some essential steps in the derivation of (2.8) and (2.9), (2.10) is a consequence of Lemma 2.1. Because of the scale invariance we can set $D=\operatorname{diag}(1-e-d, 1-e, 1)$ without loss of generality Let $\Gamma^{\prime}=\left(g_{i j}\right)_{1 \leqq i, j \leqq 3}$. Then

$$
B=\left(b_{i j}\right)=\Gamma^{\prime} A \Gamma=\left(\begin{array}{lll}
1-\alpha g_{12}^{2}-\beta g_{13}^{2} & \\
-\alpha g_{12} g_{22}-\beta g_{13} g_{23} & 1-\alpha g_{22}^{2}-\beta g_{23}^{2} & \\
-\alpha g_{12} g_{32}-\beta g_{13} g_{33} & -\alpha g_{22} g_{32}-\beta g_{23} g_{33} & 1-\alpha g_{32}^{2}-\beta g_{33}^{2}
\end{array}\right),
$$

and 


$$
T_{\Gamma} D T_{\Gamma}^{\prime}=\left(\begin{array}{ll}
(1-e-d) b_{11} & \\
(1-e-d) b_{21} & -d b_{21}^{2} / b_{11}+(1-e) b_{22} \\
(1-e-d) b_{31} & -d b_{21} b_{31} / b_{11}+(1-e) b_{32}-d b_{31}^{2} / b_{11}-e v+b_{33}
\end{array}\right)
$$

where

$$
\begin{aligned}
v & =\left(b_{31} b_{32}\right)\left(\begin{array}{ll}
b_{11} & b_{21} \\
b_{21} & b_{22}
\end{array}\right)^{-1}\left(\begin{array}{l}
b_{31} \\
b_{32}
\end{array}\right) \\
& =\frac{b_{31}^{2} b_{22}+b_{32}^{2} b_{11}-2 b_{31} b_{32} b_{21}}{b_{11} b_{22}-b_{21}^{2}} \\
& =\frac{g_{32}^{2} g_{33}^{2}(\alpha-\beta)^{2}+g_{31}^{2} g_{32}^{2} \alpha^{2}(1-\beta)+g_{31}^{2} g_{33}^{2} \beta^{2}(1-\alpha)}{1-\alpha\left(1-g_{32}^{2}\right)-\beta\left(1-g_{33}^{2}\right)+\alpha \beta g_{31}^{2}}
\end{aligned}
$$

We used the fact

$$
g_{31}^{2}=\Delta_{31}^{2}=\left(g_{12} g_{23}-g_{22} g_{13}\right)^{2}
$$

because $\left(g_{i j}\right)=\Gamma^{\prime}=\Gamma^{-1}=\left(\Delta_{i j}\right) /|\Gamma|=\left(\Delta_{i j}\right)$. Now the denominator of $v$ can be written as follows and then can be expanded in an infinite series.

$$
\begin{aligned}
1- & \alpha\left(1-g_{32}^{2}\right)-\beta\left(1-g_{33}^{2}\right)+\alpha \beta g_{31}^{2} \\
= & (1-\alpha)\left(1-\beta g_{31}^{2}-\frac{\beta-\alpha}{1-\alpha} g_{32}^{2}\right) .
\end{aligned}
$$

Similarly $1 / b_{11}=1 /\left(1-\alpha g_{12}^{2}-\beta g_{13}^{2}\right)$ can be expanded. To evaluate the integral we use the fact that $\left(g_{11}^{2}, g_{12}^{2}, g_{13}^{2}\right)$ is distributed according to the Dirichlet distribution with parameters $(1 / 2,1 / 2,1 / 2)$. One more thing needed is the following expectation $\int g_{11} g_{12} g_{21} g_{22} d \Gamma$. This can be evaluated by noting $\left(g_{11} g_{21}+g_{12} g_{22}\right)^{2}=g_{13}^{2} g_{23}^{2}$ and hence $2 \int g_{11} g_{12} g_{21} g_{22} d \Gamma=-\int g_{11}^{2} g_{21}^{2} d \Gamma$.

Now we discuss Monte Carlo methods to calculate $\hat{\Sigma}_{0}$ for general dimensionality. One objection to the estimator $\hat{\Sigma}_{0}$ might be that it is expensive to compute for large $p$. However as mentioned in Section 3 our Monte Carlo results show that the size of the replications to obtain the estimator need not be too large (at least from the viewpoint of improved risk). For $p=5,50$ replications practically achieves the same risk as $\hat{\Sigma}_{0}$. Also note that there is a subtle problem in the application of Monte Carlo method: (i) either we apply (2.5) directly for $A$, (ii), or we apply (2.5) to the roots of $A$. From logical point of view (i) is legitimate. Finite averaging itself improves the constant risk minimax estimator. Therefore for the purpose of risk comparison this method was used for the case $p=5$ and $\Sigma=I$. On the other hand the orthogonal invariance does not exactly hold with simulated uniform distribution. From practical viewpoint, however, the latter seems to be a reasonable thing to do. 
Acknowledgement. I wish to thank T.W. Anderson and C. Stein for their valuable suggestions. This research was supported in part by Office of Naval Research Contract N00014-75-C-0442 and U.S. Army Research Office Contract DAAG29-82-K-0156.

\title{
References
}

[1] Anderson, T. W., Asymptotic theory for principal component analysis. Ann. Math. Statist. 34 (1963), 122-148.

[2] Eaton, M. L., Some problems in covariance estimation. Tech. Report. No. 49, Stanford University, 1970.

[3] Efron, B. and Morris, C., Multivariate empirical Bayes and estimation of covariance matrices. Ann. Statist. 4 (1976), 22-32.

[4] Haff, L. R., Minimax extimators for a multivariate precision matrix. J. Multivariate Anal. 7 (1977), 374-385.

[5] - Estimation of the inverse covariance matrix; Random mixtures of the inverse Wishart matrix and the identity. Ann. Statist. 7 (1979a), 1264-1276.

[6] —, An identity for the Wishart distribution with applications. J. Multivariate Anal. 9 (1979b), 531-544.

[7] - Empirical Bayes estimation of the multivariate normal covariance matrix. Ann. Statist. 8 (1980), 586-597.

[8] James, W. and Stein, C., Estimation with quadratic loss. In Fourth Berkeley Symp. Math. Statist. Probability. Univ. of California Press, Berkeley, 1961.

[9] Lehmann, E. L., Testing Statistical Hypotheses, Wiley, New York, 1959.

[10] Marshall, A. W. and Olkin, I., Inequalities: Theory of majorization and its applications. Academic Press, New York, 1979.

[11] Olkin, I. and Selliah, J., Estimating covariance in a multivariate normal distribution. in Statistical Decision Theory and Related Topics II (1977), 313-326.

[12] Selliah, J., Estimation and testing problems in a Wishart distribution. Ph. D. thesis, Dept. of statistics, Stanford University, 1964.

[13] Sharma, D., An estimator of normal covariance matrix. Calcutta Statist. Assoc. Bulletin, 29 (1980), 161-167.

[14] Stein, C., Some problems in multivariate analysis, Part I. Tech. Report. No. 6, Stanford University, 1956.

[15] —, Rietz lecture. 38th annual meeting IMS. Atlanta, Georgia, 1975.

[16] Sugiura, N. and Fujimoto, M., Asymptotic risk comparison of improved estimators for normal covariance matrix. Tsukuba J. Math. 6 (1982), 103-126.

[17] Van der Vaart, H.R., On certain characteristics of the distribution of the latent roots of a symmetric random matrix under general conditions. Ann. Math. Statist. 32 (1961), 864-873.

\author{
Statistics Department, \\ Purdue University, \\ West Lafayette, IN 47907 , \\ U.S. A.
}

